\title{
Erratum
}

\section{Awareness and Use of Nutrition Information Predict Measured and Self-Rated Diet Quality of Older Adults in the United States - ERRATUM}

\author{
Anna Vaudin*, Edwina Wambogo, Alanna Moshfegh and Nadine R Sahyoun
}

doi:10.1017/S1368980020004681, Published online by Cambridge University Press, 18 November 2020

During production of the above mentioned article, the superscript values of Table 2 were erroneously modified. The correct

version is presented below.

Cambridge University Press apologise for this error.

Original:

Table 2 Nutrition awareness and use of nutrition information by socio-demographic and economic characteristics, and OR showing associations of nutrition awareness and use of nutrition information with these characteristics, among adults aged 60 years and above (n 3056), NHANES 2009-2014*

\begin{tabular}{|c|c|c|c|}
\hline \multirow[b]{2}{*}{ Characteristics } & $\begin{array}{c}\text { Nutrition } \\
\text { awareness (yes), } \\
\text { information use (yes) } \\
(n 261)\end{array}$ & $\begin{array}{c}\text { Nutrition } \\
\text { awareness (yes), } \\
\text { information use (no) } \\
(n 727)\end{array}$ & $\begin{array}{c}\text { Nutrition } \\
\text { awareness (no), } \\
\text { information use (no) } \\
(n \text { 2068) }\end{array}$ \\
\hline & \multicolumn{3}{|c|}{ Weighted row \% } \\
\hline \multicolumn{4}{|l|}{ Gender } \\
\hline Men & $5.9 \dagger$ & $27 \cdot 8$ & 66.3† \\
\hline Women & $13 \cdot 7 \dagger$ & $31 \cdot 0$ & $55 \cdot 2 \dagger$ \\
\hline \multicolumn{4}{|l|}{ Age group } \\
\hline $\begin{array}{l}\geq 76 \text { years } \\
60-75 \text { years }\end{array}$ & $\begin{array}{r}6.7 \dagger \\
11.3 \dagger\end{array}$ & $\begin{array}{l}23.9 \dagger \\
31.4 \dagger\end{array}$ & $\begin{array}{l}69.4 \dagger \\
57.3 \dagger\end{array}$ \\
\hline \multicolumn{4}{|l|}{ Education } \\
\hline$\leq$ High school/GED & $6.5 \dagger$ & $20 \cdot 1 \dagger$ & $73.4 \dagger$ \\
\hline \multirow{2}{*}{\multicolumn{4}{|c|}{ Employment }} \\
\hline & & & \\
\hline Unemployed & $10 \cdot 6$ & $25 \cdot 9 \dagger$ & $63.5 \dagger$ \\
\hline Working at a job/business & $9 \cdot 0$ & $38 \cdot 3 \dagger$ & $52 \cdot 7 \dagger$ \\
\hline \multicolumn{4}{|l|}{ Marital status } \\
\hline Single, divorced, widowed & $10 \cdot 3$ & $26 \cdot 5$ & $63 \cdot 1$ \\
\hline Married/partnered & $10 \cdot 0$ & $31 \cdot 2$ & $58 \cdot 7$ \\
\hline \multicolumn{4}{|l|}{ Race/ethnicity } \\
\hline Non-Hispanic White & $10 \cdot 9 \dagger$ & $32.5 \dagger$ & $56 \cdot 7 \dagger$ \\
\hline Non-Hispanic Black & 8.6† & $18 \cdot 4 \dagger$ & 73.0† \\
\hline Mexican American & 4.3† & $15 \cdot 8 \dagger$ & $79.9 \dagger$ \\
\hline Others & $8 \cdot 3 \dagger$ & $22 \cdot 0 \dagger$ & 69.7† \\
\hline \multicolumn{4}{|l|}{ Monthly poverty level index } \\
\hline$\leq 1.85$ & $7.5 \dagger$ & $23.5 \dagger$ & $69 \cdot 0 \dagger$ \\
\hline$>1.85$ & $11.8 \dagger$ & $33 \cdot 0 \dagger$ & $55 \cdot 2 \dagger$ \\
\hline \multicolumn{4}{|c|}{ Received cash assistance from a state or county welfare programme } \\
\hline Yes & $13 \cdot 0$ & $23 \cdot 1$ & 63.9 \\
\hline No & $10 \cdot 1$ & 29.9 & $60 \cdot 0$ \\
\hline \multicolumn{4}{|l|}{ Household food security } \\
\hline Low and very low food security & $5 \cdot 8 \dagger$ & $21.0 \dagger$ & $73 \cdot 2 \dagger$ \\
\hline Marginal food security & $8 \cdot 7 \dagger$ & $17 \cdot 6 \dagger$ & $73 \cdot 7 \dagger$ \\
\hline Full food security & $10 \cdot 7 \dagger$ & $31.0 \dagger$ & $58 \cdot 3 \dagger$ \\
\hline
\end{tabular}


Table 2 Continued

\begin{tabular}{|c|c|c|c|}
\hline \multirow[b]{2}{*}{ Characteristics } & $\begin{array}{c}\text { Nutrition } \\
\text { awareness (yes), } \\
\text { information use (yes) } \\
(n 261)\end{array}$ & $\begin{array}{c}\text { Nutrition } \\
\text { awareness (yes), } \\
\text { information use (no) } \\
(n 727)\end{array}$ & $\begin{array}{c}\text { Nutrition } \\
\text { awareness (no), } \\
\text { information use (no) } \\
(n \text { 2068) }\end{array}$ \\
\hline & \multicolumn{3}{|c|}{ Weighted row \% } \\
\hline \multicolumn{4}{|c|}{ Emergency food last 12 months } \\
\hline Yes & $8 \cdot 6$ & $16 \cdot 7 \dagger$ & $74 \cdot 7 \dagger$ \\
\hline No & $10 \cdot 3$ & $30 \cdot 3 \dagger$ & $59 \cdot 4 \dagger$ \\
\hline \multicolumn{4}{|c|}{ Home-delivered meals last 12 months } \\
\hline Yes & 6.5 & $19 \cdot 8$ & $73.6 \dagger$ \\
\hline No & $10 \cdot 3$ & 29.9 & $59.9 \dagger$ \\
\hline \multicolumn{4}{|c|}{ Congregate meals last 12 months } \\
\hline Yes & $10 \cdot 7$ & $22 \cdot 5$ & $66 \cdot 8$ \\
\hline No & $10 \cdot 1$ & $30 \cdot 0$ & 59.9 \\
\hline \multicolumn{4}{|c|}{ Received SNAP last 12 months } \\
\hline Yes & $7 \cdot 1$ & $21.5 \dagger$ & $71.4 \dagger$ \\
\hline No & 10.5 & $30 \cdot 6+$ & $58.9+$ \\
\hline \multicolumn{4}{|l|}{ On special diet } \\
\hline Yes & $17.9 \dagger$ & $26 \cdot 9$ & $55 \cdot 2$ \\
\hline No & $9 \cdot 0 \dagger$ & $30 \cdot 7$ & $60 \cdot 3$ \\
\hline \multicolumn{4}{|l|}{ Self-rated health } \\
\hline Excellent/very good & $13.0+$ & $36 \cdot 3+$ & $50 \cdot 7 \dagger$ \\
\hline Good & $9.5 \dagger$ & $27 \cdot 3 \dagger$ & $63 \cdot 2 \dagger$ \\
\hline Fair/poor & $5.8 \dagger$ & $21.0 \dagger$ & $73 \cdot 2 \dagger$ \\
\hline \multicolumn{4}{|l|}{ Self-rated diet } \\
\hline Excellent/very good & $15 \cdot 0 \dagger$ & $30 \cdot 1$ & $54.9 \dagger$ \\
\hline Good & $8.2 \dagger$ & $30 \cdot 0$ & $61.8+$ \\
\hline Fair/poor & $2.6 \dagger$ & $27 \cdot 1$ & $70 \cdot 3 \dagger$ \\
\hline
\end{tabular}

NHANES, National Health and Nutrition Examination Survey; GED, General Education Development; SNAP, Supplemental Nutrition Assistance Program.

${ }^{*}$ Significant difference in proportions tested using $\chi^{2}$ test for categorical variables, percentages across the row may not sum to 100 due to rounding.

†Percentages within a column with unlike superscript letters were significantly different $(P<0.05)$.

\section{Correction:}

Table 2 Nutrition awareness and use of nutrition information by socio-demographic and economic characteristics, and OR showing associations of nutrition awareness and use of nutrition information with these characteristics, among adults aged 60 years and above (n 3056), NHANES 2009-2014*

\begin{tabular}{|c|c|c|c|}
\hline \multirow[b]{2}{*}{ Characteristics } & $\begin{array}{c}\text { Nutrition } \\
\text { awareness (yes), } \\
\text { information use (yes) } \\
(n 261)\end{array}$ & $\begin{array}{c}\text { Nutrition } \\
\text { awareness (yes), } \\
\text { information use (no) } \\
(n 727)\end{array}$ & $\begin{array}{c}\text { Nutrition } \\
\text { awareness (no), } \\
\text { information use (no) } \\
(n \text { 2068) }\end{array}$ \\
\hline & \multicolumn{3}{|c|}{ Weighted row \% } \\
\hline \multicolumn{4}{|l|}{ Gender } \\
\hline Men & $5.9^{a}$ & $27 \cdot 8$ & $66 \cdot 3^{a}$ \\
\hline Women & $13 \cdot 7^{b}$ & $31 \cdot 0$ & $55 \cdot 2^{b}$ \\
\hline \multicolumn{4}{|l|}{ Age group } \\
\hline$\geq 76$ years & $6 \cdot 7^{\mathrm{a}}$ & $23.9^{a}$ & $69 \cdot 4^{\mathrm{a}}$ \\
\hline$\overline{60}-75$ years & $11 \cdot 3^{b}$ & $31.4^{b}$ & $57 \cdot 3^{b}$ \\
\hline \multicolumn{4}{|l|}{ Education } \\
\hline$\leq$ High school/GED & $6 \cdot 5^{a}$ & $20 \cdot 1^{a}$ & $73.4^{\mathrm{a}}$ \\
\hline Some college/college + & $12 \cdot 7^{b}$ & $36 \cdot 0^{b}$ & $51.4^{b}$ \\
\hline \multicolumn{4}{|l|}{ Employment } \\
\hline Unemployed & $10 \cdot 6$ & $25 \cdot 9^{a}$ & $63.5^{\mathrm{a}}$ \\
\hline Working at a job/business & $9 \cdot 0$ & $38 \cdot 3^{b}$ & $52 \cdot 7^{b}$ \\
\hline \multicolumn{4}{|l|}{ Marital status } \\
\hline Single, divorced, widowed & $10 \cdot 3$ & $26 \cdot 5$ & $63 \cdot 1$ \\
\hline Married/partnered & $10 \cdot 0$ & $31 \cdot 2$ & $58 \cdot 7$ \\
\hline \multicolumn{4}{|l|}{ Race/ethnicity } \\
\hline Non-Hispanic White & $10 \cdot 9^{a}$ & $32.5^{a}$ & $56 \cdot 7^{\mathrm{a}}$ \\
\hline Non-Hispanic Black & $8 \cdot 6^{\mathrm{a}}$ & $18 \cdot 4^{\mathrm{b}}$ & $73 \cdot 0^{\mathrm{b}, \mathrm{c}}$ \\
\hline Mexican American & $4 \cdot 3^{b}$ & $15 \cdot 8^{b}$ & $79 \cdot 9^{c}$ \\
\hline Others & $8 \cdot 3^{a, b}$ & $22 \cdot 0^{b}$ & $69 \cdot 7^{b, d}$ \\
\hline
\end{tabular}


Table 2 Continued

\begin{tabular}{|c|c|c|c|}
\hline \multirow[b]{2}{*}{ Characteristics } & $\begin{array}{c}\text { Nutrition } \\
\text { awareness (yes), } \\
\text { information use (yes) } \\
(n 261)\end{array}$ & $\begin{array}{c}\text { Nutrition } \\
\text { awareness (yes), } \\
\text { information use (no) } \\
(n 727)\end{array}$ & $\begin{array}{c}\text { Nutrition } \\
\text { awareness (no), } \\
\text { information use (no) } \\
(n \text { 2068) }\end{array}$ \\
\hline & \multicolumn{3}{|c|}{ Weighted row \% } \\
\hline \multicolumn{4}{|l|}{ Monthly poverty level index } \\
\hline$\leq 1.85$ & $7.5^{a}$ & $23.5^{a}$ & $69 \cdot 0^{a}$ \\
\hline$>1.85$ & $11 \cdot 8^{b}$ & $33.0^{b}$ & $55 \cdot 2^{b}$ \\
\hline \multicolumn{4}{|c|}{ Received cash assistance from a state or county welfare programme } \\
\hline Yes & $13 \cdot 0$ & $23 \cdot 1$ & 63.9 \\
\hline No & $10 \cdot 1$ & 29.9 & $60 \cdot 0$ \\
\hline \multicolumn{4}{|l|}{ Household food security } \\
\hline Low and very low food security & $5 \cdot 8^{\mathrm{a}}$ & $21 \cdot 0^{a}$ & $73.2^{a}$ \\
\hline Marginal food security & $8.7^{\mathrm{a}, \mathrm{b}}$ & $17 \cdot 6^{\mathrm{a}}$ & $73.7^{a}$ \\
\hline Full food security & $10 \cdot 7^{\mathrm{b}}$ & $31.0^{b}$ & $58 \cdot 3^{b}$ \\
\hline \multicolumn{4}{|l|}{ Emergency food last 12 months } \\
\hline Yes & $8 \cdot 6$ & $16 \cdot 7^{\mathrm{a}}$ & $74.7^{\mathrm{a}}$ \\
\hline No & $10 \cdot 3$ & $30 \cdot 3^{b}$ & $59 \cdot 4^{\mathrm{b}}$ \\
\hline \multicolumn{4}{|c|}{ Home-delivered meals last 12 months } \\
\hline Yes & 6.5 & $19 \cdot 8$ & $73 \cdot 6^{a}$ \\
\hline No & $10 \cdot 3$ & 29.9 & $59.9^{b}$ \\
\hline \multicolumn{4}{|l|}{ Congregate meals last 12 months } \\
\hline Yes & $10 \cdot 7$ & $22 \cdot 5$ & $66 \cdot 8$ \\
\hline No & $10 \cdot 1$ & $30 \cdot 0$ & 59.9 \\
\hline \multicolumn{4}{|l|}{ Received SNAP last 12 months } \\
\hline Yes & $7 \cdot 1$ & $21.5^{a}$ & $71.4^{a}$ \\
\hline No & 10.5 & $30 \cdot 6^{b}$ & $58.9^{b}$ \\
\hline \multicolumn{4}{|l|}{ On special diet } \\
\hline Yes & $17 \cdot 9^{a}$ & $26 \cdot 9$ & $55 \cdot 2$ \\
\hline No & $9 \cdot 0^{\mathrm{b}}$ & $30 \cdot 7$ & $60 \cdot \overline{3}$ \\
\hline \multicolumn{4}{|l|}{ Self-rated health } \\
\hline Excellent/very good & $13 \cdot 0^{\mathrm{a}}$ & $36 \cdot 3^{a}$ & $50 \cdot 7^{\mathrm{a}}$ \\
\hline Good & $9.5^{a}$ & $27 \cdot 3^{b}$ & $63 \cdot 2^{b}$ \\
\hline Fair/poor & $5 \cdot 8^{b}$ & $21 \cdot 0^{c}$ & $73 \cdot 2^{c}$ \\
\hline \multicolumn{4}{|l|}{ Self-rated diet } \\
\hline Excellent/very good & $15 \cdot 0^{\mathrm{a}}$ & $30 \cdot 1$ & $54 \cdot 9^{a}$ \\
\hline Good & $8 \cdot 2^{b}$ & $30 \cdot 0$ & $61 \cdot 8^{b}$ \\
\hline Fair/poor & $2 \cdot 6^{\mathrm{c}}$ & $27 \cdot 1$ & $70 \cdot 3^{c}$ \\
\hline
\end{tabular}

NHANES, National Health and Nutrition Examination Survey; GED, General Education Development; SNAP, Supplemental Nutrition Assistance Program. a,b,c,dPercentages within a column with unlike superscript letters were significantly different $(P<0.05)$.

*Significant difference in proportions tested using $\chi^{2}$ test for categorical variables, percentages across the row may not sum to 100 due to rounding.

\section{Reference}

Vaudin, A., Wambogo, E., Moshfegh, A., \& Sahyoun, N. (2020). Awareness and use of nutrition information predict measured and self-rated diet quality of older adults in the USA. Public Health Nutrition 24, 1687-1697. doi: 10.1017/S1368980020004681 